OPEN ACCESS

Edited by:

Francisco Lopez-Munoz, Camilo José Cela University, Spain

Reviewed by:

Dennis Kusters,

University of Michigan, United States Rodrigo Azevedo Loiola

Artois University, France Hengli Tian,

Shanghai Jiao Tong University, China

*Correspondence:

Xiaochuan Sun

sunxch1445@qq.com

Zhijian Huang

zhijian@cqmu.edu.cn;

526997993@qq.com

†These authors have contributed equally to this work

Specialty section:

This article was submitted to

Neuropharmacology,

a section of the journal

Frontiers in Neuroscience

Received: 08 November 2020 Accepted: 15 January 2021

Published: 18 February 2021

Citation:

Liu H, He J, Wu Y, Du Y, Jiang Y,

Chen C, Yu Z, Zhong J, Wang Z, Cheng C, Sun $X$ and Huang $Z$ (2021) Endothelial Regulation by Exogenous Annexin A1 in Inflammatory Response and BBB Integrity Following Traumatic

Brain Injury

Front. Neurosci. 15:627110. doi: 10.3389/fnins.2021.627110

\section{Endothelial Regulation by Exogenous Annexin A1 in Inflammatory Response and BBB Integrity Following Traumatic Brain Injury}

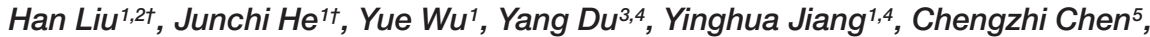 \\ Zhanyang Yu ${ }^{4}$, Jianjun Zhong ${ }^{1}$, Zhigang Wang ${ }^{2}$, Chongjie Cheng ${ }^{1}$, Xiaochuan Sun ${ }^{1 *}$ and \\ Zhijian Huang ${ }^{1 *}$

\begin{abstract}
'Department of Neurosurgery, The First Affiliated Hospital of Chongqing Medical University, Chongqing, China, ${ }^{2}$ Department of Neurosurgery, Qilu Hospital of Shandong University (Qingdao Campus), Qingdao, China, ${ }^{3}$ Department of Neurology, Xiangya Hospital, Central South University, Changsha, China, ${ }^{4}$ Departments of Radiology and Neurology, Massachusetts General Hospital, Harvard Medical School, Boston, MA, United States, ${ }^{5}$ Department of Occupational and Environmental Health, School of Public Health and Management, Research Center for Medicine and Social Development, Innovation Center for Social Risk Governance in Health, Chongqing Medical University, Chongqing, China
\end{abstract}

Background and Target: Following brain trauma, blood-brain barrier (BBB) disruption and inflammatory response are critical pathological steps contributing to secondary injury, leading to high mortality and morbidity. Both pathologies are closely associated with endothelial remodeling. In the present study, we concentrated on annexin A1 (ANXA1) as a novel regulator of endothelial function after traumatic brain injury.

Methods: After establishing controlled cortical impact (CCl) model in male mice, human recombinant ANXA1 (rANXA1) was administered intravenously, followed by assessments of BBB integrity, brain edema, inflammatory response, and neurological deficits.

Result: Animals treated with rANXA1 $(1 \mu \mathrm{g} / \mathrm{kg})$ at $1 \mathrm{~h}$ after $\mathrm{CCl}$ exhibited optimal BBB protection including alleviated BBB disruption and brain edema, as well as endothelial junction proteins loss. The infiltrated neutrophils and inflammatory cytokines were suppressed by rANXA1, consistent with decreased adhesive and transmigrating molecules from isolated microvessels. Moreover, rANXA1 attenuated the neurological deficits induced by $\mathrm{CCl}$. We further found that the Ras homolog gene family member A (RhoA) inhibition has similar effect as rANXA1 in ameliorating brain injuries after $\mathrm{CCl}$, whereas rANXA1 suppressed CCl-induced RhoA activation.

Conclusion: Our findings suggest that the endothelial remodeling by exogenous rANXA1 corrects BBB disruption and inflammatory response through RhoA inhibition, hence improving functional outcomes in $\mathrm{CCl}$ mice.

Keywords: annexin A1, blood-brain barrier, inflammation, RhoA, traumatic brain injury 


\section{BACKGROUND}

Traumatic brain injury (TBI) has been a major cause of death and disability worldwide for decades (Wu et al., 2020). Although the mortality rate of patients with severe TBI has decreased by nearly $50 \%$ in the past 150 years, it is still as high as 30\% (Stein et al., 2010). More than 30 TBI clinical trials have failed until now (Wright et al., 2014; Delbary-Gossart et al., 2016), and there is currently no effective treatment for TBI. Therefore, the research on TBI treatment strategy and prognosis has become the latest hotspot. Following initial impact, the secondary inflammatory response, blood-brain barrier (BBB) breakdown, and edema formation contribute to the clinical deterioration in patients, and the interplay between them has also been recognized. Robust inflammation can damage the endothelial junctions of normal BBB, and then BBB leakage permits movement of water from vasculature to the extracellular space in response to elevated osmotic gradient generated by the leakage of vascular components into the brain parenchyma, leading to vasogenic edema (Donkin and Vink, 2010). As a direct consequence of BBB leakage, brain edema is responsible for $50 \%$ of patients' clinical deterioration following TBI (Feickert et al., 1999; Marmarou., 2003).

Annexin A1 (ANXA1) is dominantly expressed in the endothelium of the brain microvasculature and colocalizes with cellular cytoskeleton (Cristante et al., 2013). The BBB consists of tight and adherent junction complexes between endothelial cells. Thus, endothelial tight junction proteins (such as claudin5, occludin, and ZO-1) and endothelial adherent proteins such as VE cadherin are essential to BBB integrity (Zhang et al., 2019). It was proven that ANXA1 is capable of regulating physiological and multiple sclerosis-conditioned BBB integrity and promoting cytoskeletal stability and enhancing endothelial junction protein expression by inhibiting the Ras homolog gene family member A (RhoA) (Cristante et al., 2013). RhoA is known to initiate the signaling pathway, resulting in the destabilization of the actin cytoskeleton, ultimately enhancing paracellular permeability (Terry et al., 2010). As a major downstream effector of RhoA, Rho-associated coiled-coil kinase (ROCK) regulates actin reorganization during cell adhesion, migration, contraction, and proliferation (Liu et al., 2018). On the other hand, ANXA1 acted to augment rolling velocity and reduce neutrophil adhesion to endothelium (Girol et al., 2013). ANXA1-null mice exhibited a higher extent of neutrophil extravasation, which is also associated with RhoA activity (Rao et al., 2017). Both pathological events are closely related to endothelial function; thus, we proposed a therapeutic potential of ANXA1 as an early regulator of vascular remodeling in TBI, putatively with the involvement of RhoA.

\section{METHODS}

\section{Animals and Controlled Cortical Impact Model}

All animal procedures were conducted in compliance with the Animal Research: Reporting In Vivo Experiments (ARRIVE) guidelines-the National Institutes of Health Guide for the Care and Use of Laboratory Animals. All animal experiments were approved by the Animal Care and Use Committee of Chongqing Medical University.

Mice were 12 weeks old and weighed 20-22 g. The sham group received only craniotomy without cortical impact. Briefly, after deep isoflurane (3\%) anesthesia, a midline longitudinal incision was performed; the skin was retracted to expose the skull. After establishing controlled cortical impact (CCI) model in male mice, a 5.0-mm-diameter craniotomy was established in the right parietal bone midway between bregma and lambda with the medial edge $1.0 \mathrm{~mm}$ lateral to the midline. Mice were impacted at $5.0 \mathrm{~m} / \mathrm{s}$ with a $40-\mathrm{ms}$ dwell time and $0.6-\mathrm{mm}$ depression using a 3.0-mm-diameter convex tip, mimicking a moderate brain trauma in humans. Following surgery, bone wax was used to fill the hole on the skull, and the scalp was sutured closed. The animals were on an electric blanket to reduce mice suffering. They were held in an electric blanket to maintain body temperature until complete recovery from anesthesia. Then, human recombinant ANXA1 (rANXA1) (R\&D, United States) was administrated through the tail vein after CCI. ROCK inhibitor Y27632 (20 nmol/kg, cytoskeleton) was injected into the contralateral ventricle $(1 \mathrm{~mm}$ lateral and $0.5 \mathrm{~mm}$ backward to bregma on the skull surface) at $30 \mathrm{~min}$ pre-CCI (Huang et al., 2012).

\section{BBB Leakage}

The integrity of $\mathrm{BBB}$ was reflected by Evans blue in the brain hemisphere following CCI. First, Evans blue dye (2\% in saline, $4 \mathrm{~mL} / \mathrm{kg}$ ) was injected through tail vein $1 \mathrm{~h}$ before being sacrificed. Under deep anesthesia, the chest wall was opened, and mice were perfused with phosphate-buffered saline (PBS) intracardially. After decapitation, brain hemispheres were removed, weighed, and homogenized in trichloroacetic acid and centrifuged at 14,000 rpm for $15 \mathrm{~min}$. Then, the resultant supernatants were separated and analyzed at $630 \mathrm{~nm}$ for excitation and $680 \mathrm{~nm}$ for emission using a spectrophotometer (SpectraMax M5). The Evans blue amount was quantified according to a linear standard curve.

The quantification of extravasated immunoglobulin G (IgG) molecules was determined by immunohistochemical staining, modified from the previous way (Zhang et al., 2016; Thomas et al., 2020). Brain slices $500 \mu \mathrm{m}$ apart (total nine sections in each animal to cover lesion cavity) were blocked with $5 \%$ donkey serum in PBS with $0.05 \%$ Tween 20 for $1 \mathrm{~h}$. Brain slices were incubated with Biotin-SP AffiniPure $\mathrm{F}\left(\mathrm{ab}^{\prime}\right) 2$ Fragment Donkey Anti-Mouse IgG $(\mathrm{H}+\mathrm{L})$ antibody (1:200; Jackson Immunoresearch Laboratories, United States) at $4^{\circ} \mathrm{C}$ overnight, followed by reaction with fluorescein streptavidin (1:200, Jackson Immunoresearch Laboratories, United States). The IgG immunointensity in injured hemisphere of each level was calculated by ImageJ software and added as final value, while contralateral hemisphere as negative control. The IgG-positive area was observed under fluorescence microscope.

\section{Water Content}

These specimens were dried in an oven at $100^{\circ} \mathrm{C}$ for $48 \mathrm{~h}$. The following formula was used to calculate the percentage of 
water content in hemispheres: ([wet weight-dry weight]/wet weight) $\times 100 \%$.

\section{Immunohistochemistry}

First, coronal brain slices above were fixed with $4 \%$ paraformaldehyde and then blocked with 5\% donkey serum for $60 \mathrm{~min}$. After incubation overnight at $4^{\circ} \mathrm{C}$ with rat antineutrophil (1:200, Abcam), slides were scanned with fluorescence microscope (ECLIPSE Ti-s, Nikon, Japan). For quantification, the number of neutrophil cells per $20 \times$ field in the perilesion area was counted in four randomized fields (two in cortex and two in subcortex) of each animal. And the immunoreactivity of IgG area per $20 \times$ field in the perilesion area was calculated in four randomized fields (two in cortex and two in subcortex) in each animal.

\section{Immunoblots}

Brain tissue dissected from injured cortex within the injured hemisphere or from the left hemisphere of uninjured control mice was homogenized on ice in RIPA (radioimmunoprecipitation assay) buffer (Beyotime, China) containing protease inhibitor cocktail (Sigma-Aldrich). Homogenates were centrifuged at 14,000 revolutions $/ \mathrm{min}$ (rpm) for $15 \mathrm{~min}$ at $4^{\circ} \mathrm{C}$. Protein content of the supernatant was assayed (Bio-Rad Laboratories, United States), and aliquots of protein were boiled in denaturing sample buffer (62.5 mmol/L Tris $\mathrm{pH}$ 6.8, 2\% sodium dodecyl sulfate, $5 \mathrm{mmol} / \mathrm{L}$ ethylenediaminetetraacetic acid, 10\% glycerol, $0.25 \%$ 2-mercaptoethanol, $0.01 \%$ bromophenol blue). The protein samples were denatured by boiling and were resolved $(20 \mu \mathrm{g})$ on 6 or $10 \%$ sodium dodecyl sulfate-polyacrylamide gels (Invitrogen) and blotted onto polyvinylidene difluoride membranes (Millipore). GAPDH was used as an internal reference. Membranes were blocked for $1 \mathrm{~h}$ in $5 \%$ milk in Tris-buffered saline ( $\mathrm{pH} 7.4$ ) containing $0.1 \%$ Tween 20 and then incubated overnight at $4^{\circ} \mathrm{C}$ with primary antibodies: GAPDH (Cell Signaling, 1:1,000), ANXA1 (Abcam, 1:500), ZO-1 (Santa Cruz, 1:1,000), occludin (Santa Cruz, 1:1,000), VE cadherin (Santa Cruz, 1:1,000), claudin-5 (Abcam, 1:1,000), RhoA (Santa Cruz, 1:2,000), vascular cell adhesion molecule 1 (VCAM-1) (Santa Cruz, 1:1,000), intercellular adhesion molecule 1 (ICAM-1) (Santa Cruz, 1:1,000), E-selectin (Santa Cruz, 1:1,000), and CD99 (Abcam, 1:1,000). Membranes were washed in Tris-buffered saline Tween 20 and then incubated for $1 \mathrm{~h}$ with an appropriate horseradish peroxidase-conjugated secondary antibody (1:20,000 in Tris-buffered saline Tween 20,5\% milk) at room temperature. Proteins of interest were detected using the enhanced chemiluminescence kit (Beyotime, China) and filmed. The optical densities for protein bands were analyzed quantified with Quantity One 4.6.2 (Bio-Rad Laboratories, United States). Three independent experiments were carried out to verify protein expression.

\section{RhoA Activation Assay}

GTP-RhoA and total-RhoA were extracted from injured cortex tissue according to the instruction of Rho Activation Assay Kits (Cytoskeleton, United States). For total RhoA expression,
$40 \mu \mathrm{g}$ of total protein was used. Activated/total RhoA was detected using an anti-RhoA antibody (Cytoskeleton). RhoA activity semiquantification was determined by using ImageJ and expressed as the ratio between RhoA/total RhoA. Results were expressed as a relative ratio, normalized to the mean value of the sham group.

\section{Enzyme-Linked Immunosorbent Assay}

After harvest of injured cortex tissue, the homogenate was centrifuged at $5,000 \times g$ for $5 \mathrm{~min}$. The supernatant was collected and prepared for subsequent assay. Interleukin-1 $\beta$ (IL-1 $\beta$ ) and tumor necrosis factor $\alpha$ (TNF- $\alpha)$ concentrations were measured using commercially available enzyme-linked immunosorbent assay (ELISA) kits (USCN Life Science, China) according to the protocols of the kits. Briefly, the samples were added to each well of monoclonal anti-mouse IL- $1 \beta$ or TNF- $\alpha$ antibody-coated microtiter plates (ELISA plates) for $30 \mathrm{~min}$ at $37^{\circ} \mathrm{C}$. Unbound material was washed off, and bound antibody was detected by addition of horseradish peroxidase for $15 \mathrm{~min}$ at $37^{\circ} \mathrm{C}$. Absorbance was measured $15 \mathrm{~min}$ after addition of substrate. A standard curve was constructed using various dilutions of IL- $1 \beta$ and TNF- $\alpha$.

\section{Microvessel Isolation}

Microvessel isolation was conducted as previously described (Corvol et al., 2018). The mice were sacrificed $24 \mathrm{~h}$ after injury. After perfusion with PBS, the brains were harvested. Two sides of hemispheres (contralateral side and ipsilateral side) were separated; the core lesion areas (which fall apart quite easily) and undermined white matter were removed, rolling on the filter paper to get rid of meninges and the remaining loose debris. After this, cortical tissue from each hemisphere was pooled, homogenized in cold PBS on ice, and then centrifuged at $4^{\circ} \mathrm{C}$, $1,000 \times g$ for $5 \mathrm{~min}$. The tissue pellet was suspended in PBS and then centrifuged again at $4^{\circ} \mathrm{C}, 1,500 \times g$ for $20 \mathrm{~min}$. The resuspended pellet with PBS was filtered with cell strainer $(70 \mu \mathrm{m})$ to get rid of the debris. The extracted tissue from six mice merged into each sample.

\section{Behavioral Tests}

Before and after the CCI model (days -1, 1, 3, 7, 14, and 28), the neurological functions of the mice were evaluated using a set of neurobehavioral tasks [neurological severity score (NSS)]. Briefly, NSS test was carried out as Tsenter's protocol (Tsenter et al., 2008). A ten-point NSS was applied. The neuroscore consisted of 10 different tasks evaluating motor ability, alertness, balancing, and general behavior. One point was awarded for failure to complete a task.

Furthermore, the Morris water maze was applied to evaluate cognitive function from 15 to 21 days post-CCI as previously described (Huang et al., 2016). Briefly, five consecutive daily training sessions were performed for learning latency. For each trial, mice were randomized to start from one of four directions (north, south, east, or west) to find the submerged platform within $90 \mathrm{~s}$. Mice that failed to reach the platform within $90 \mathrm{~s}$ were placed on the platform by the researcher and allowed to remain there for $15 \mathrm{~s}$. In the probe trial, staying period and entry times 
of the platform area and target quadrant were recorded at day 21. To exclude the potential difference of visual ability between groups, extra visible trial was performed using a labeled platform above the water level. The behavior assessments as mentioned previously were performed by an independent investigator who was blind to the group assignment.

\section{Lesion Volume}

At 28 days after CCI, the animals were perfused with $0.1 \mathrm{~mol} / \mathrm{L}$ PBS under deep anesthesia. Brains were frozen-sectioned at the thickness of $10 \mu \mathrm{m}$. Brain slices $500 \mu \mathrm{m}$ apart were stained with hematoxylin-eosin then photographed. The target level was between $1 \mathrm{~mm}$ anterior and $3 \mathrm{~mm}$ posterior to the bregma. Brain slices $500 \mu \mathrm{m}$ apart (total nine sections in each animal) were collected to cover the core lesion area. The volume of injured tissue was measured with ImageJ 1.48 software. Damaged tissue volume $=$ contralateral hemisphere volume-ipsilateral hemisphere volume.

\section{Statistical Analysis}

Data are presented as mean $\pm \mathrm{SD}$. The generated data were normally distributed (Zhao et al., 2017). All the analyses were performed with the SigmaStat software (San Jose, CA, United States). Immunoblot and immunohistochemistry data were analyzed by one-way analysis of variance (ANOVA) followed by Tukey-Kramer post hoc tests. Neurobehavioral assessments were analyzed by repeated-measures ANOVA (Tornero et al., 2013; Zemmar et al., 2014; Delbary-Gossart et al., 2016), followed by Tukey post hoc tests. One-way ANOVA followed by post hoc test was used to evaluate the statistical differences in each time point between groups. The most conservative multiple-test correction was applied using the Bonferroni method. For all analyses, $p<0.05$ was considered significant.

\section{RESULTS}

\section{ANXA1 Expression Increased in the Perilesion Cortex After TBI}

To verify the endogenous reaction of ANXA1 to brain trauma, we measured its expression in the injured hemisphere of CCI mice at different time points (Figure 1A). The immunoblots showed a delayed increase of the ANXA1 level after injury. Compared with the sham-operated animals, there was a 1.7-fold increase of ANXA1 expression at day 1 after CCI $(p<0.05)$, and the elevation peaked at day $3(p<0.05)$. After that, the expression declined and returned to baseline at day 14 .

\section{Effects of rANXA1 Administration on BBB Disruption, Neurological Deficits, and Lesion Size After $\mathbf{C C l}$}

To assess the precise and optimal effects of ANXA1 on BBB permeability after CCI, we examined Evans blue extravasation in bilateral hemispheres at $24 \mathrm{~h}$ after CCI with different timing (1, 3 , and 5 h post-surgery, Figure 1B) and graded dose (1, 5, and
$10 \mu \mathrm{g} / \mathrm{kg}$ ) of rANXA1 administration (Figure 1C). After CCI, the leakage of Evans blue was detected in the ipsilateral hemispheres. Our results showed that rANXA1 treatment $(1 \mu \mathrm{g} / \mathrm{kg})$ at 1,3 , and $5 \mathrm{~h}$ post-CCI significantly ameliorated dye extravasation compared to the vehicle group $(p<0.05)$ and $1 \mathrm{~h}$ post-CCI protocol induced the least decrease in Evans blue amount. We thus chose $1 \mathrm{~h}$ post-CCI as the optimal time point for drug administration. We applied serial doses of rANXA1 ranging from 1 to $10 \mu \mathrm{g} / \mathrm{kg}$, and the results show that each dose was efficient to prevent Evans blue leakage $(p<0.05)$. Because the initial dose $(1 \mu \mathrm{g} / \mathrm{kg})$ seemed to exert significant effect in BBB protection, and further increase in concentration failed to enhance its efficiency, this administrative setting $(1 \mu \mathrm{g} / \mathrm{kg}$ at $1 \mathrm{~h}$ post-CCI) was selected for subsequent experiments.

To determine the gross effects of human rANXA1 on the functional recovery after CCI, we compared the performances of animals in NSS and Morris water maze tests (Figures 1D-F). After CCI, the neurological impairments were observed in all the tests. Consistently, the animals treated with rANXA1 exhibited significant overall improvement in these examinations compared with vehicle group $(p<0.05)$. To examine if rANXA1 contributes to the brain tissue damage after CCI, the volume of lesion cavity was measured at 28 days after CCI (Figure 1G). Compared with the vehicle-treated mice, rANXA1 failed to significantly change the lesion volume after CCI $(p>0.05)$.

\section{Effects of rANXA1 Administration on BBB Permeability and Brain Edema After $\mathbf{C C I}$}

To learn the dynamic and persistent bioactivity from rANXA1 in $\mathrm{BBB}$ protection, similar experiments were repeated at 24 and $72 \mathrm{~h}$ after CCI separately (Figure 2A). After rANXA1 treatment, less extravasated dye was detected in injured hemisphere until $72 \mathrm{~h}$ following CCI $(p<0.05)$. Given that BBB damage may lead to vasogenic cerebral edema, we next assessed the ability of rANXA1 at post-traumatic brain water content (Figure 2B). Following $\mathrm{CCI}$, the water content in the vehicle group increased remarkably in the ipsilateral hemisphere. This disruption was attenuated by rANXA1 treatment with significance $(p<0.05)$.

The spatial profile of BBB protection by rANXA1 was additionally measured at $24 \mathrm{~h}$ after CCI. Under fluorescence microscope, plasma-derived IgG leakage was observed in the ipsilateral cortex, striatum, and hippocampus following CCI, whereas the contralateral hemisphere was comparatively normal (Figure 2C). And rANXA1 treatment significantly reduced IgG extravasation area as compared to vehicle (Figure 2C).

\section{Effects of rANXA1 Administration on Junctional Proteins After $\mathbf{C C l}$}

To elucidate the structural alteration of BBB following CCI, the integrity of tight and adherent junction was further assessed. The expressions of junctional proteins (Occludin, claudin-5, ZO1 , and VE cadherin) were significantly reduced at $24 \mathrm{~h}$ postCCI $(p<0.05)$ (Figure 3). However, rANXA1 preserved the expression levels of occludin, ZO-1, and VE-cadherin $(p<0.05)$, rather than claudin-5 $(p>0.05)$. 
A

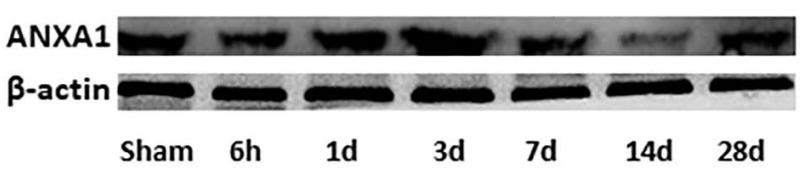

B

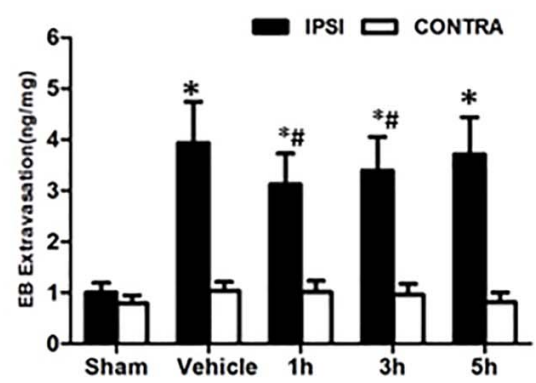

D

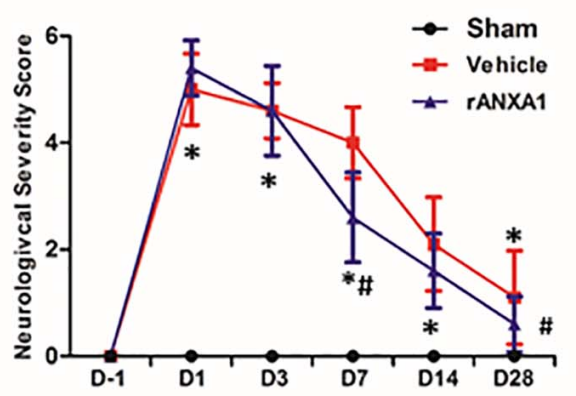

F
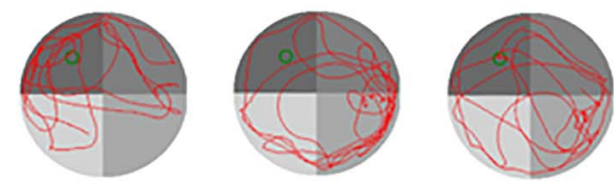

Sham

Vehicle

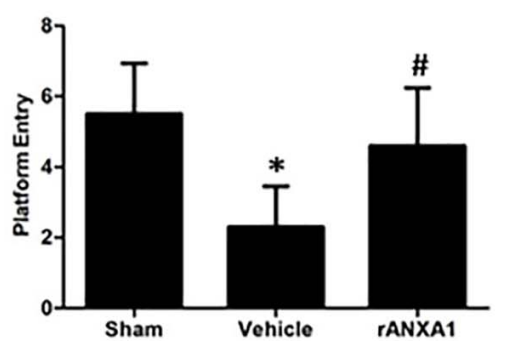

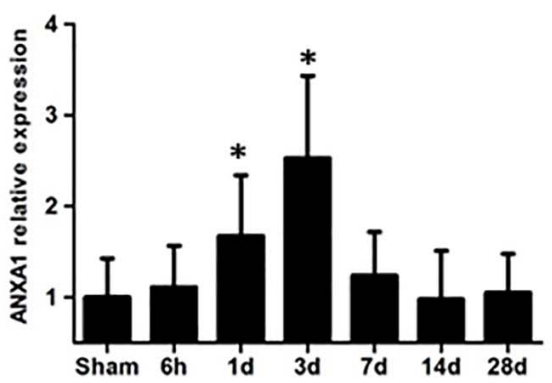

C

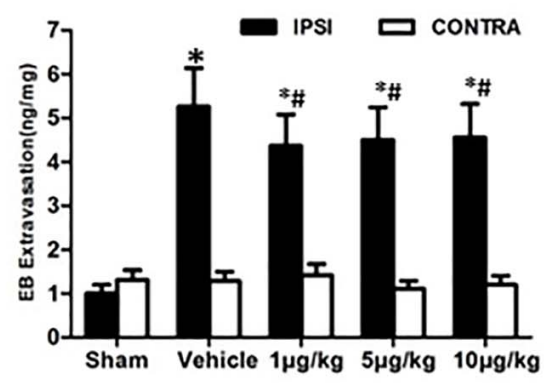

E

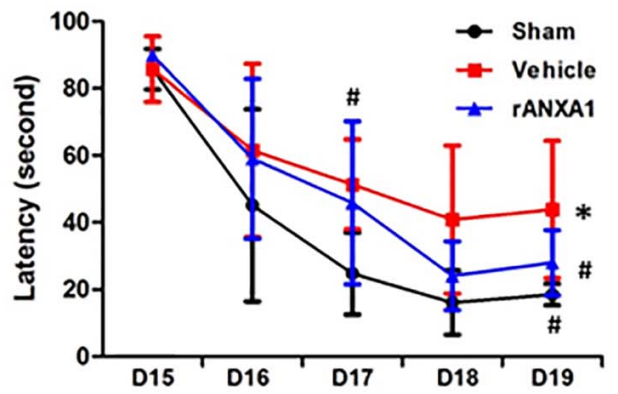

G

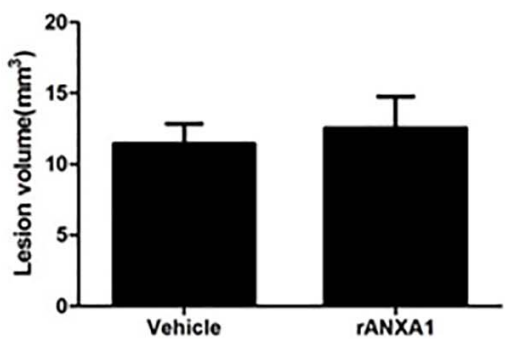

FIGURE 1 | Effects of rANXA1 administration on BBB integrity after CCI. (A) Temporal expression of ANXA1 after CCI. ANXA1 expression increased at day 1 after $\mathrm{CCl}(p<0.05)$ and the elevation peaked at day $3(p<0.05)$. After that, the expression declined and returned to baseline at day 14 . Representative bands and quantitative analysis of endogenous ANXA1 expression in the ipsilateral cortex. Relative density of each protein has been normalized against the sham group. ${ }^{*} p<0.05$ vs. sham, $n=5$ per group. (B,C) Effects of rANXA1 administration on BBB integrity after CCl. rANXA1 treatment $1 \mathrm{~h}$ post-CCl protocol induced more decrease in Evans blue amount than $3 \mathrm{~h}$ post-CCl. Therefore, $1 \mathrm{~h}$ post-CCl was chosen as the optimal time point for drug administration in the following study. Vehicle group was treated $1 \mathrm{~h}$ post-CCl (B). Serial doses of rANXA1 ranging from 1 to $10 \mu \mathrm{g} / \mathrm{kg}$ were efficient to prevent Evans blue leakage $(p<0.05)$. ${ }^{*} p<0.05$ vs. sham, $\# p<0.05$ vs. vehicle, $n=8$ per group. Effects of rANXA1 on functional outcomes and lesion size after CCl (D-G). The animals treated with rANXA1 exhibited significant overall improvement in these examinations compared with vehicle group $(p<0.05)$. Compared with the vehicle-treated mice, rANXA1 failed to change the lesion volume significantly after $\mathrm{CCl}(p>0.05)$. Neurological severity scores before and after $\mathrm{CCl}(\mathbf{D})$. Learning latency $(\mathbf{E})$ and probe trial $(\mathbf{F})$ in Morris water maze after CCl. (G) Lesion volume at 28 days after CCl. ${ }^{*} p<0.05$ vs. sham, $\# p<0.05$ vs. vehicle, $n=10$ per group. 
A

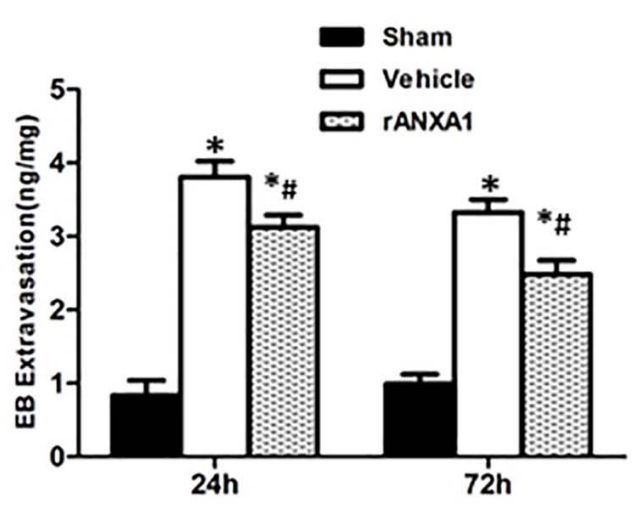

B

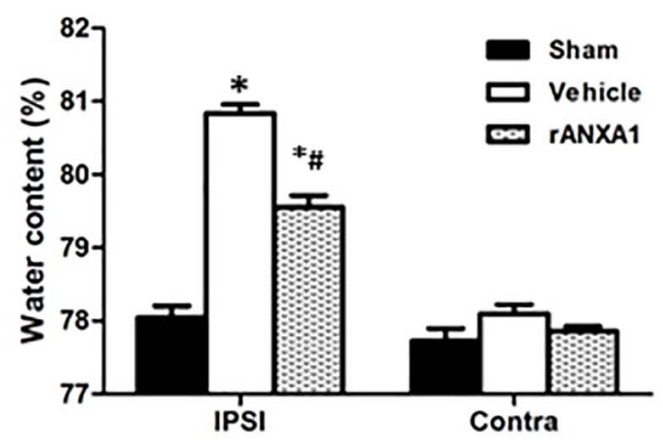

C

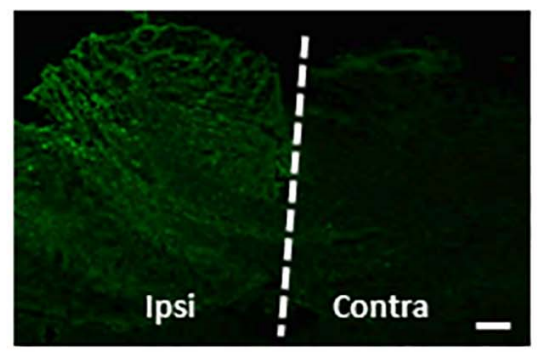

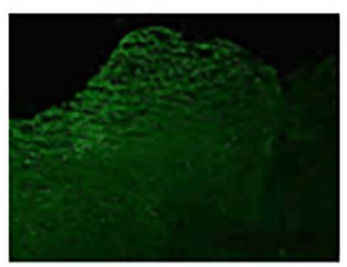

Vehicle

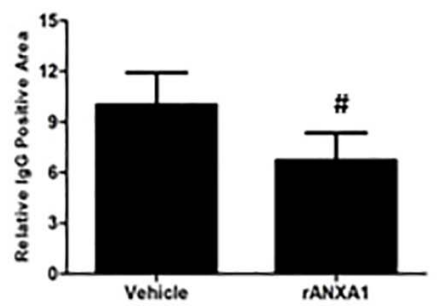

FIGURE 2 | Effects of rANXA1 on BBB disruption after CCl. (A) Quantitation of extravasated Evans blue at 24 and $72 \mathrm{~h}$ after CCl and (B) quantitation of hemispherical water content at $24 \mathrm{~h}$ after $\mathrm{CCl}$. After rANXA1 treatment, less extravasated dye was detected in injured hemisphere until $72 \mathrm{~h}$ following $\mathrm{CCl}$ ( $p<0.05$ ). Following $\mathrm{CCl}$, the water content in the vehicle group increased remarkably in ipsilateral hemisphere. This disruption was attenuated by rANXA1 treatment with significance $(p<0.05)$. ${ }^{*} p<0.05$ vs. sham, $\# p<0.05$ vs. vehicle, $n=8$ per group. (C) Effects of rANXA1 on BBB disruption after CCl. Representative images and quantitative analysis of IgG fluorescence surrounding the lesion area at $24 \mathrm{~h}$ after CCl. Under fluorescence microscope, plasma-derived lgG leakage was observed in the ipsilateral cortex, striatum, and hippocampus, whereas the contralateral hemisphere was comparatively normal. rANXA1 treatment significantly reduced lgG extravasation area as compared to vehicle. Relative immunoactivity was normalized to the sham group. ${ }^{\star} p<0.05$ vs. sham, $\# p<0.05$ vs. vehicle, $n=5$ per group. Scale bar $=100 \mu \mathrm{m}$.

\section{Effects of rANXA1 Administration on Inflammatory Response and Endothelial Molecules After CCI}

To assess the potential effects of rANXA1 on inflammatory response, we measured neutrophil counts in injured cortex at $72 \mathrm{~h}$ in mice pretreated with rANXA1 or vehicle (Figure 4A). It showed that brain neutrophil number surrounding the lesion area significantly decreased after rANXA1 treatment $(p<0.05)$. In addition, we actually measured the temporal changes of neutrophil infiltration on the preinjury day and days 1, 3, and 7 post-CCI (Supplementary Figure 1). We almost did not see any infiltrated neutrophil in the sham group. And the results also showed that brain neutrophil number surrounding the lesion area increased maximally on day $3(p<0.05)$. Moreover, we examined the protein levels of classical proinflammatory cytokines at $72 \mathrm{~h}$ post-CCI by ELISA (Figure 4B). It showed that rANXA1 significantly reduced the expression of IL-1 $\beta$ and TNF- $\alpha$ compared to the vehicle group $(p<0.05)$.
To explore the possible mechanism involved in ANXA1associated anti-inflammation effect, the microvessels were isolated to analyze the expression of endothelial molecules at $72 \mathrm{~h}$ post-CCI (Figure 4C). CCI resulted in a remarkable increase in VCAM-1, ICAM-1, and E-selectin expressions $(p<0.05)$. The protein levels of VCAM-1 and ICAM-1 were significantly declined after rANXA1 treatment $(p<0.05)$. It did not induce any statistical difference in E-selectin expression $(p>0.05)$. Moreover, rANXA1 significantly suppressed the expression of CD99 within endothelial cells $(p<0.05)$.

\section{ANXA1 Treatment Inhibited RhoA Activity and Thereby Modulated Endothelial Function}

To verify the signaling pathway potentiating ANXA1-driven BBB protection, the RhoA activation (GTP-RhoA/RhoA ratio) was investigated before and after CCI (Figure 4D). The expression 

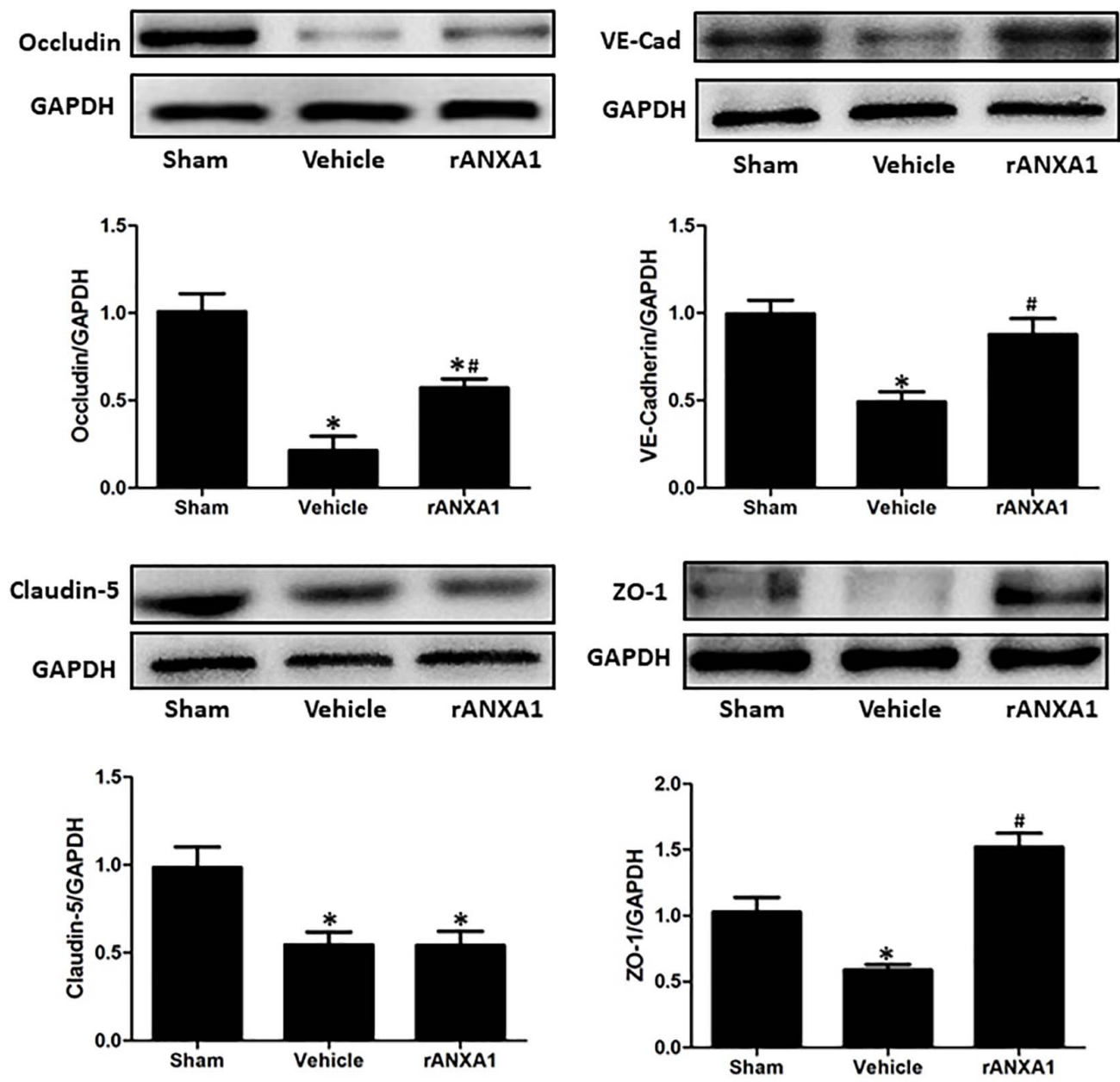

FIGURE 3 | Effects of rANXA1 on the expressions of endothelial junctional proteins after CCI. The expressions of junctional proteins (occludin, claudin-5, ZO-1, and VE-cadherin) were significantly reduced at $24 \mathrm{~h}$ post-CCI $(p<0.05)$. However, rANXA1 preserved the expression levels of occludin, ZO-1, and VE-cadherin $(p<0.05)$, rather than claudin-5 ( $p>0.05)$. Representative bands and quantitative analysis of the expressions of junctional proteins at $24 \mathrm{~h}$ after CCl. Relative density of each protein was normalized to the sham group. ${ }^{\star} p<0.05$ vs. sham, $\# p<0.05$ vs. vehicle, $n=5$ per group.

of activated RhoA (GTP-RhoA) was enhanced at $24 \mathrm{~h}$ postCCI. And the rAXNA1 treatment significantly inhibited RhoA activation as compared to vehicle $(p<0.05)$.

The bioactivities of RhoA on endothelial function were evaluated by ROCK inhibitor Y27632 (Figures 5A,B). First, the expressions of junctional proteins were repeatedly measured, and Y27632 preserved the expression levels of occludin, ZO-1, and VE-cadherin $(p<0.05)$, rather than claudin-5 $(p>0.05)$. Then, endothelial molecules from isolated microvessels were analyzed by immunoblot. The protein levels of VCAM-1, ICAM1 , and CD99 were significantly suppressed by Y27632 treatment $(p<0.05)$.

\section{RhoA Inhibition Exhibited Similar Effects in Ameliorating Brain Injuries After CCI as rANXA1 Administration}

As demonstrated in Figures 6A,B, the Y27632 induced less extravasated dye in injured hemisphere at both 24 and $72 \mathrm{~h}$
post-CCI $(p<0.05)$ and attenuated post-traumatic brain water content with significance $(p<0.05)$. For inflammatory response, neutrophil number surrounding the lesion area was significantly decreased by Y27632 treatment ( $p<0.05)$ (Figure 6C). Furthermore, Y27632 significantly reduced protein levels of IL$1 \beta$ and TNF- $\alpha$ as compared to the vehicle group $(p<0.05)$ (Figure 6D). Finally, the functional results showed Y27632 induced the overall improvements in all the behavioral tests with significance $(p<0.05)$ (Figures 6E-G).

\section{DISCUSSION}

It is well known that endothelial dysfunction occurs right after brain injury and plays a central role in the secondary response, including BBB disruption, vasogenic edema, and inflammation. Recent studies imply the potential of vascular remodeling by ANXA1 based on the roles of ANXA1 in endothelial function (Ikebuchi and Waisman, 1990; Hubaishy et al., 1995; 
A

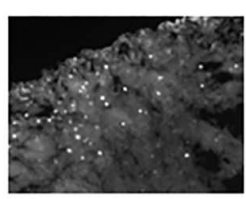

Vehicle

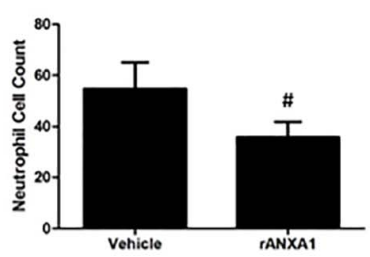

C
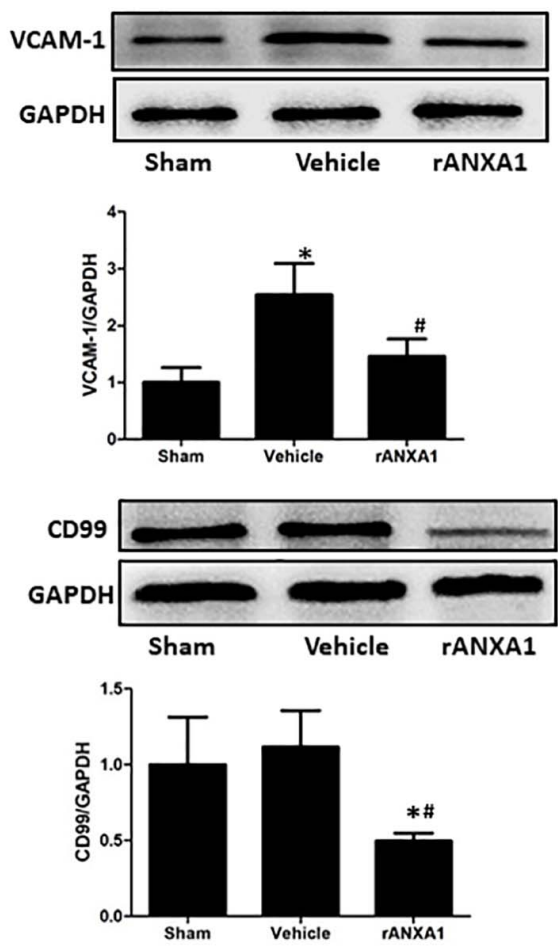

D

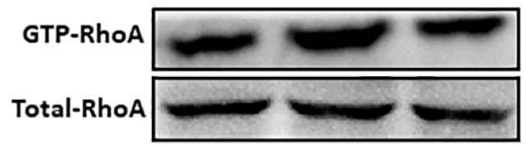

B
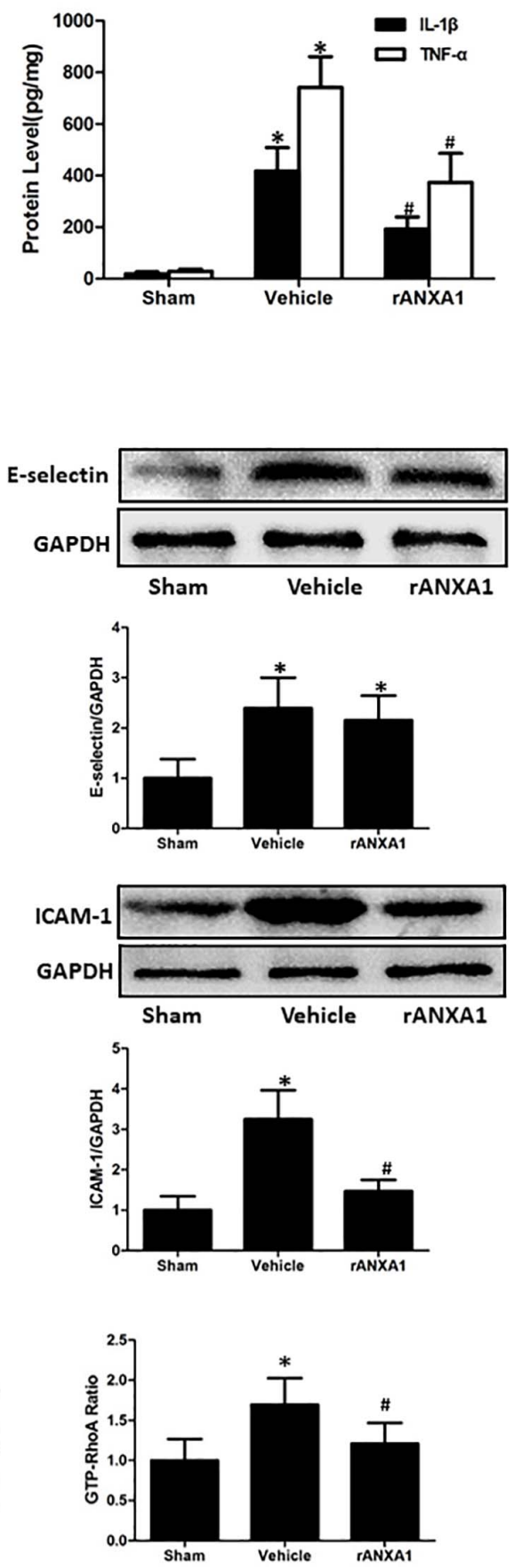

FIGURE 4 | Effects of rANXA1 on systemic infiltrating inflammation after $\mathrm{CCl}$ (A-C). (A) Representative images and quantitative analysis of neutrophil infiltration surrounding the lesion area at $72 \mathrm{~h}$ after $\mathrm{CCl}$. Brain neutrophil number surrounding the lesion area significantly decreased after $\mathrm{rANXA1}$ treatment $(p<0.05)$. (B) Quantitative analysis of the protein levels of inflammatory cytokines at $72 \mathrm{~h}$ after CCl. rANXA1 significantly reduced the expression of IL-1 $\beta$ and TNF- $\alpha$ compared to the vehicle group $(p<0.05)$. (C). Representative bands and quantitative analysis of the expressions of endothelial molecules at $72 \mathrm{~h}$ after $\mathrm{CCI}$. CCI resulted in a remarkable increase in VCAM-1, ICAM-1, and E-selectin expressions $(\rho<0.05)$. The protein levels of VCAM-1 and ICAM-1 were significantly declined after rANXA1 treatment $(p<0.05)$. It did not induce any statistical difference in E-selectin expression $(p>0.05)$. Moreover, rANXA1 significantly suppressed the expression of CD99 within endothelial cells $(p<0.05)$. (D) Effects of rANXA1 on RhoA activity after CCl. The expression of activated RhoA (GTP-RhoA) was enhanced at $24 \mathrm{~h}$ post-CCl. And the rAXNA1 treatment significantly inhibited RhoA activation as compared to vehicle $(p<0.05)$. ${ }^{*} p<0.05$ vs. sham, $\# p<0.05$ vs. vehicle, $n=8$ per group in panel (A), scale bar $=100 \mu \mathrm{m} ; n=6$ per group in panel (B); $n=5$ per group in panels (C,D). 


\section{A}
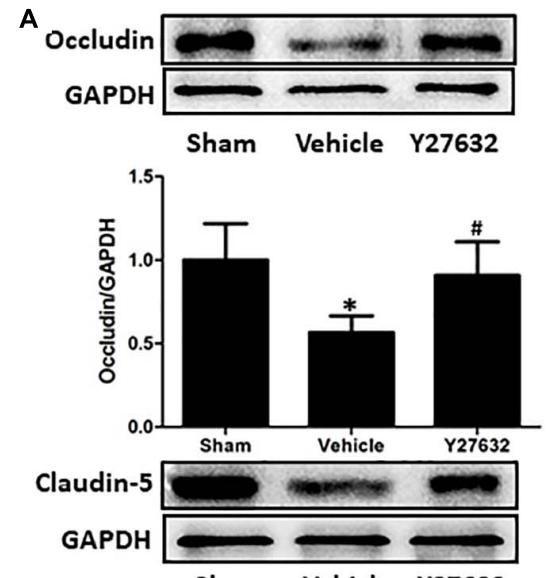

B
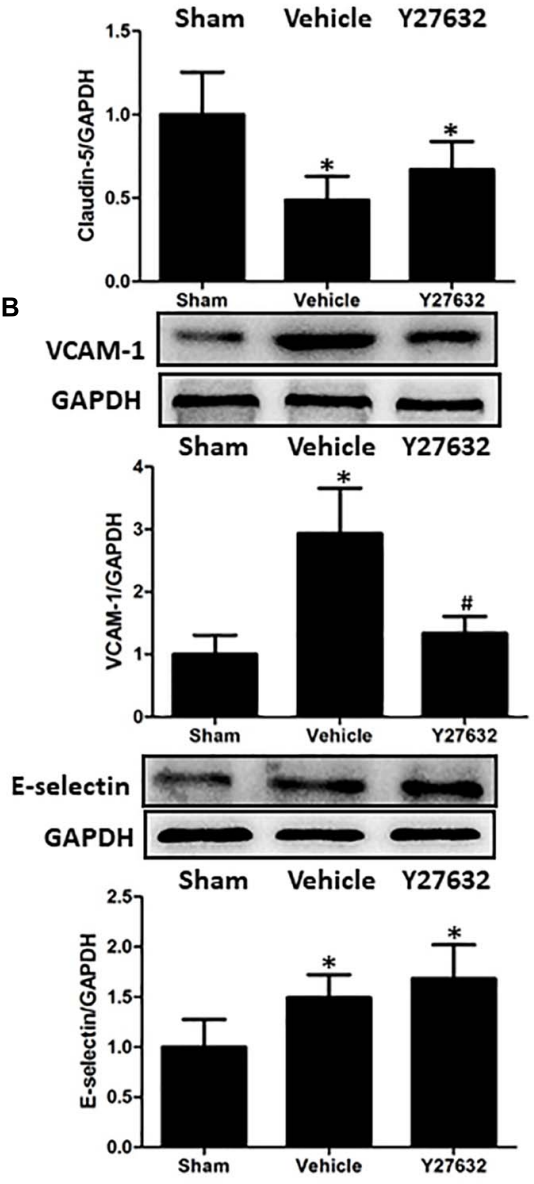

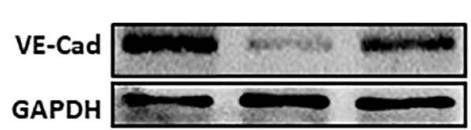

GAPDH

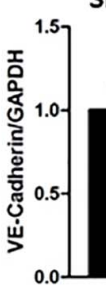

Sham Vehicle Y27632
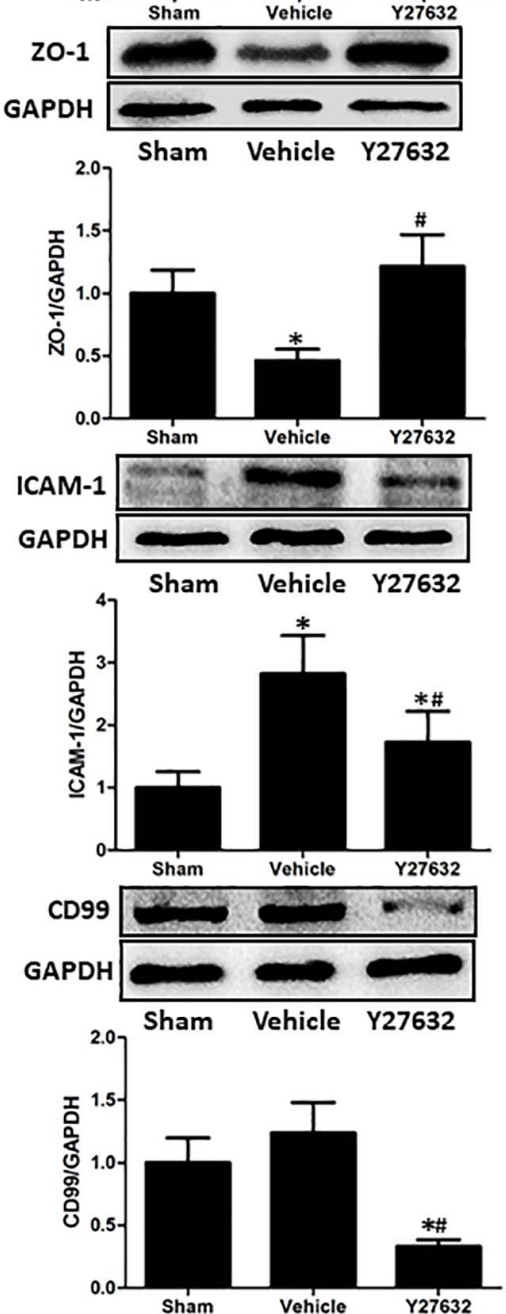

FIGURE 5 | Effects of Y27632 on endothelial function after CCl. (A) Representative bands and quantitative analysis of the expressions of occludin, VE-cadherin, claudin-5, and ZO-1 at $24 \mathrm{~h}$ after CCI. Y27632 preserved the expression levels of occludin, ZO-1, and VE-cadherin ( $p<0.05)$, rather than claudin-5 ( $p>0.05)$. (B) Representative bands and quantitative analysis of the expressions of endothelial molecules at $72 \mathrm{~h}$ after $\mathrm{CCl}$. The endothelial molecules from isolated microvessels were analyzed. The protein levels of VCAM-1, ICAM-1, and CD99 were significantly suppressed by Y27632 treatment ( $p<0.05)$. Relative density of each protein was normalized to the sham group. ${ }^{*} p<0.05$ vs. sham, $\# p<0.05$ vs. vehicle, $n=5$ per group.

Lee et al., 2004; Su et al., 2010). Therefore, we hypothesized that exogenous ANXA1 administration may stabilize BBB integrity, decreasing cerebral edema and inflammatory response following TBI. After optimization of dose and timing protocols, the $\mathrm{BBB}$ protection by ANXA1 was strongly validated, and an administration protocol of $1 \mu \mathrm{g} / \mathrm{kg}$ at $1 \mathrm{~h}$ after operation was selected. Subsequent results demonstrate that ANXA1 treatment improved the neurological outcomes following CCI, correlated with alleviated $\mathrm{BBB}$ leakage, cerebral edema, and systemic infiltrating inflammation. 
A

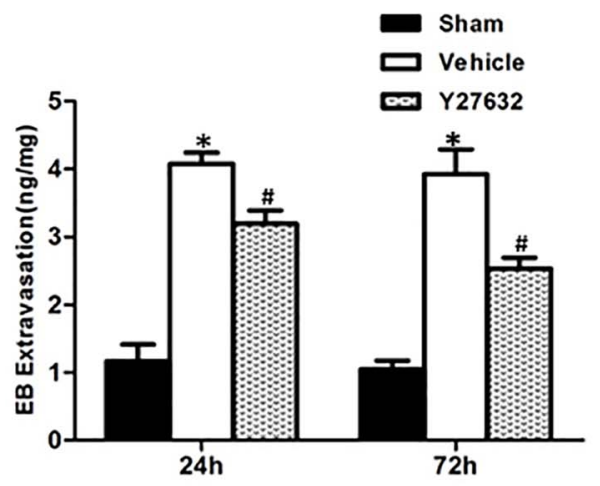

B

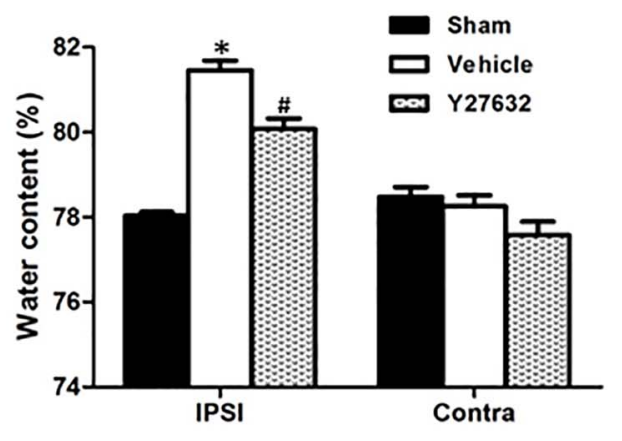

C

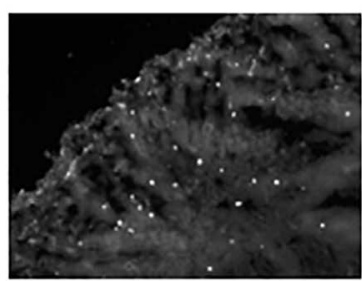

Vehicle

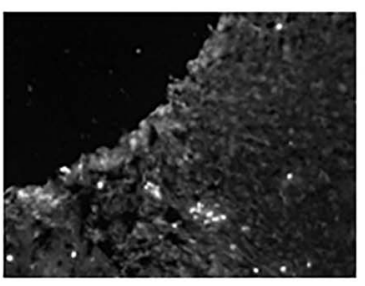

Y27632

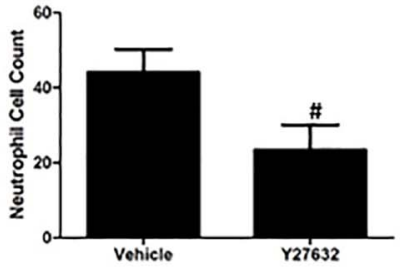

D

E
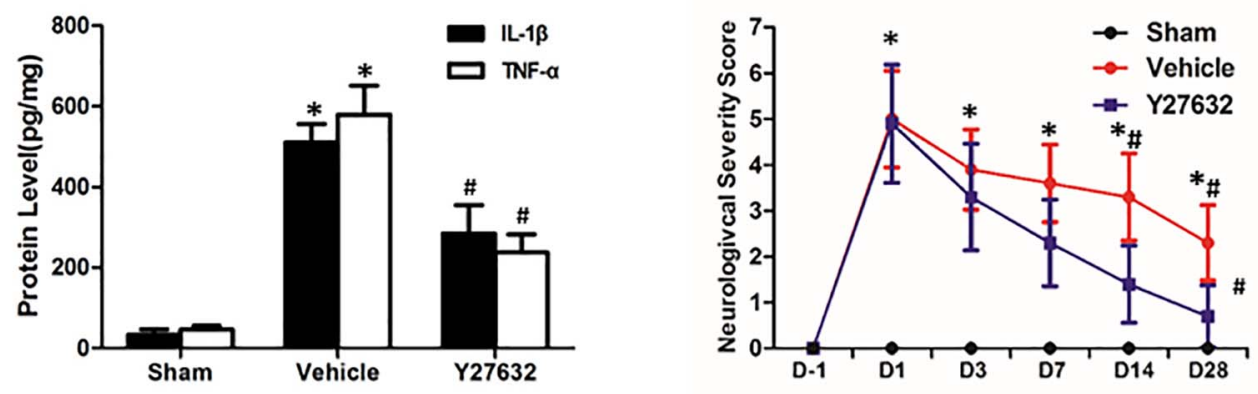

F

G

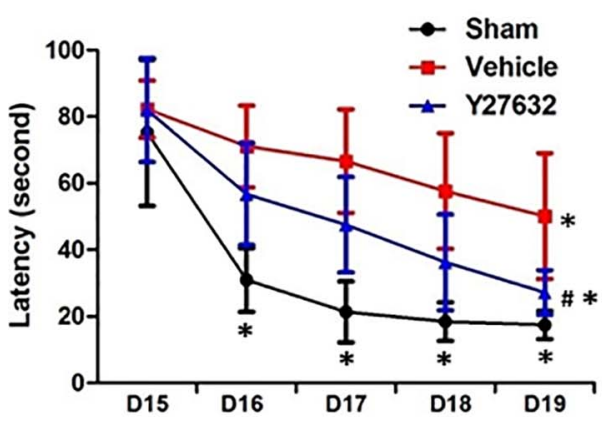

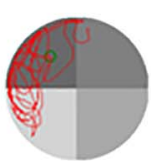

Sham

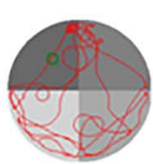

Vehicle

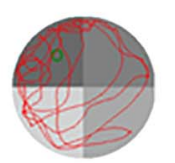

Y27632

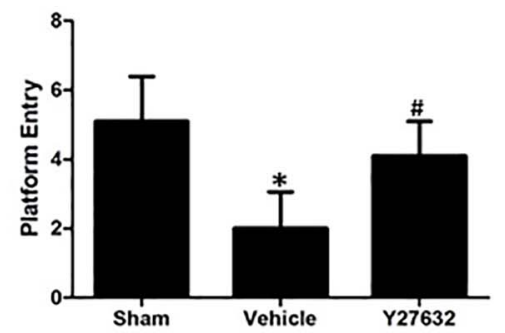

FIGURE 6 | Effects of Y27632 on secondary brain injuries after CCl. (A) Y27632 induced less extravasated dye in injured hemisphere at both 24 and $72 \mathrm{~h}$ post-CCl $(p<0.05)$. (B) Y27632 attenuated post-traumatic brain water content with significance $(p<0.05)$. (C) Neutrophil number surrounding the lesion area was significantly decreased by Y27632 treatment $(p<0.05)$. (D) Y27632 significantly reduced protein levels of IL-1 $\beta$ and TNF- $\alpha$ as compared to the vehicle group $(p<0.05)$. (E) Neurological severity scores before and after $\mathbf{C C l}$, (F) learning latency, and (G) probe trial in Morris water maze after CCl. The functional results showed Y27632 induced the overall improvements in all the behavioral tests with significance $(p<0.05)$. ${ }^{*} p<0.05$ vs. sham, \#p<0.05 vs. vehicle, $n=8$ per group

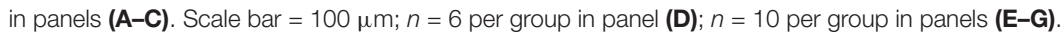


Recent studies indicated the protection of ANXA1 on BBB (McArthur et al., 2016), which is supported by our current results in CCI model. Endogenous ANXA1 response to CCI initiated until $24 \mathrm{~h}$ post-injury, whereas rANXA1 treatment at $3 \mathrm{~h}$ post$\mathrm{CCI}$ helped restore $\mathrm{BBB}$ integrity, indicating an encouraging time window for TBI treatment. Among different time frames, rANXA1 treatment at $1 \mathrm{~h}$ post-CCI induced minimal BBB leakage, highlighting the importance of early intervention. In principle, the permeability of BBB is largely based on the function of endothelial junctions (Bose et al., 2018). Thus, the benefits on $\mathrm{BBB}$ here may be derived from preserved junctional proteins by rANXA1. Following TBI, BBB disruption permits movement of water from vasculature to the extracellular space in response to elevated osmotic gradient generated by the leakage of vascular components into the brain parenchyma, leading to vasogenic edema (Donkin and Vink, 2010; Luh et al., 2019). As a direct consequence of BBB leakage, edema is responsible for $50 \%$ of patient deaths decrease following TBI (Feickert et al., 1999; Marmarou., 2003). In the present study, rANXA1 treatment induced decreased edema in ipsilateral hemispheres following CCI, consistent with the attenuated neurological impairments.

In addition to endothelial cytoskeleton, systemic infiltrating inflammation was also believed to account for BBB disruption. For example, intracerebral administration of IL-1 $\beta$ can induce BBB leakage and related vasogenic edema (Holmin and Mathiesen, 2000; Wang et al., 2020). As a hallmark of systemic infiltrating inflammation following TBI, neutrophil recruited to the inflammatory site requires adhesion to endothelial cells and then cross the blood vessel wall (Sugimoto et al., 2016), which is orchestrated by various molecules expressed by endothelial cells (Subramanian et al., 2016). For example, CD99 has been most extensively studied for its role in neutrophil transmigration, whereas other molecules, notably ICAM-1, VCAM-1, and E-selectin, were known to potentiate neutrophil adhesion (Muller, 2013). Here we showed suppressed neutrophil infiltration and inflammatory cytokines by rANXA1 treatment, partially due to the decreased adhesive and transmigrating capabilities within endothelium. In addition, a previous study also proved that ANXA1 is associated with microglia activity (Liu et al., 2016); thus, ANXA1 may influence inflammatory cytokine expression by acting directly on microglia, as well as reducing neutrophil infiltration.

As a member of Rho family of GTPases, activated RhoA is believed to increase $\mathrm{BBB}$ permeability through the destabilization of endothelial actin proteins and tight junction proteins (Terry et al., 2010). However, conflicting results from recent studies seemed to challenge our understanding on it, that local RhoA activation initiates the formation of contractile F-actin structures surrounding neutrophil-induced endothelial pores, preventing plasma leakage during neutrophil diapedesis (Heemskerk et al., 2016). Here we showed active RhoA was suppressed by rANXA1 treatment following CCI, consistent with decreased $\mathrm{BBB}$ leakage and junctional proteins loss by rANXA1, suggesting that the junctional restoration from RhoA inhibition outweighs its effect on endothelial pore formation. Furthermore, RhoA/ROCK pathway was lastly reported to affect adhesive molecules expression, and RhoA blockade ameliorated macrophage transfer to the endothelium (Huang et al., 2012). Our studies using isolated microvessels from injured hemisphere showed that RhoA acted to promote neutrophil-endothelial interaction through VCAM-1, ICAM-1, and CD99, which was negatively regulated by ANXA1.

Overall, our data demonstrated a multipotent role of rANXA1 in microvascular modulation (junctional barrier preservation,

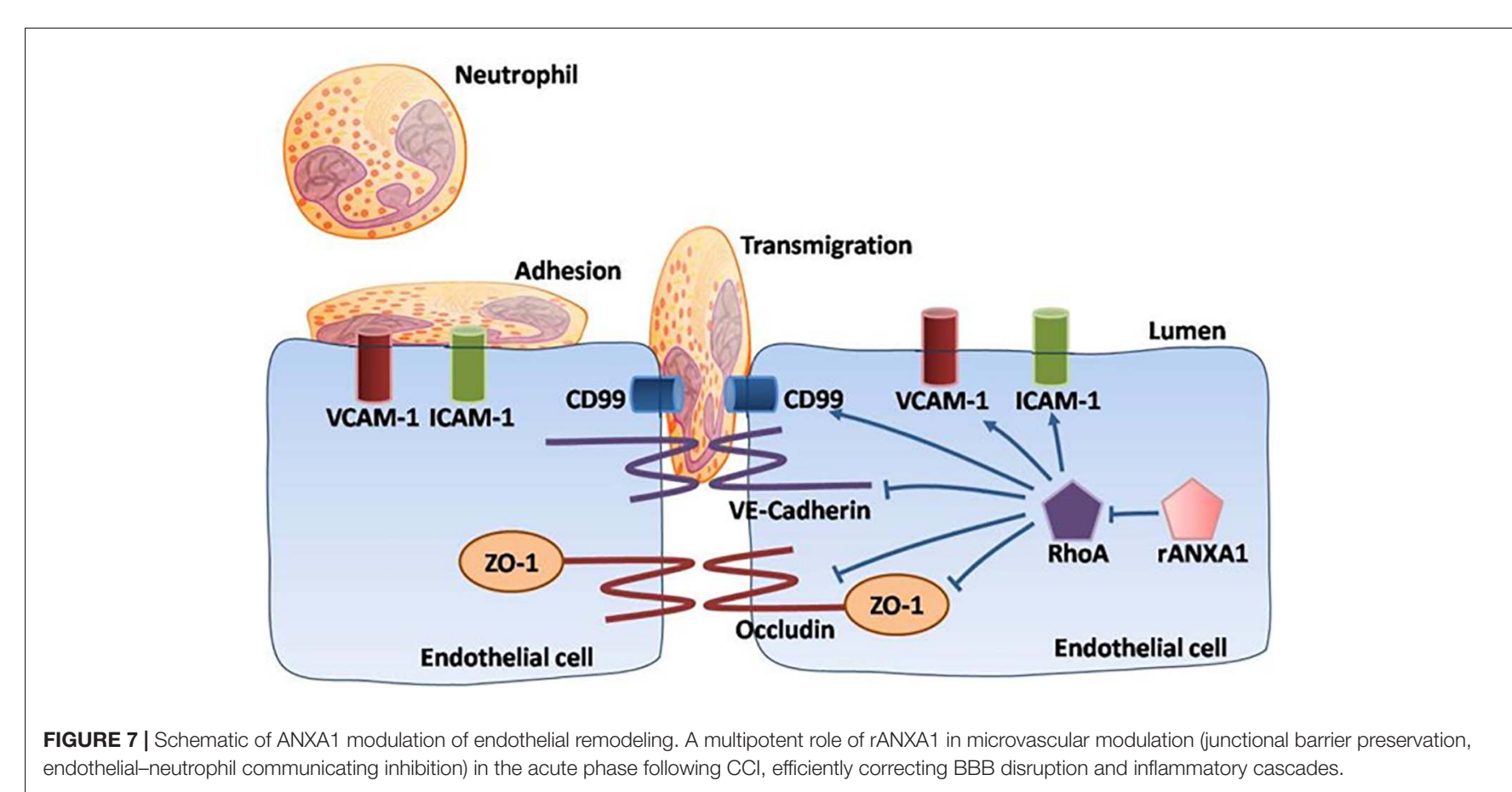


endothelial-neutrophil communicating inhibition) in the acute phase following CCI, efficiently correcting BBB disruption and inflammatory cascades (Figure 7). Moreover, rANXA1 treatment exhibited beneficial effect on neurological outcomes, but not on lesion cavity volume. It is acceptable because lesion size might be more associated with primary brain injury following trauma, so it is difficult to be changed by secondary medical intervention. The functional improvements by rANXA1 can be equally achieved by RhoA inhibition. Taken together, these findings suggest that exogenous rANXA1 inhibits BBB disruption and systemic infiltrating inflammation through RhoA inhibition, hence improving functional outcomes in CCI mice. For translational practice, our results provide a novel and practical agent with two advantages in TBI therapy, i.e., ideal administration pathway (intravenous) and effective time window ( $3 \mathrm{~h})$. It would be worthwhile to seek more potentials of ANXA1 in chronic endothelial events. In addition, cerebral hemorrhage was also considered to be closely associated with the integrity of the BBB. For example, matrix metalloproteinases have been shown to directly disrupt tight junctions, which dramatically altered water reflectance and solute permeability of the BBB, exacerbating brain edema and hemorrhagic contusion expansion (Terry et al., 2010). As the BBB protection by ANXA1 was strongly validated by our study, ANXA1 may play a role on hemorrhagic contusion expansion.

\section{CONCLUSION}

Taken together, these findings suggest that exogenous rANXA1 inhibits $\mathrm{BBB}$ disruption and systemic infiltrating inflammation through RhoA inhibition, hence improving functional outcomes in CCI mice. For translational practice, our results provide a novel and practical agent with two advantages, i.e., ideal administration pathway (intravenous) and effective time window ( $3 \mathrm{~h})$. Therefore, ANXA1 intervention could be an encouraging target in the TBI therapy.

\section{DATA AVAILABILITY STATEMENT}

The original contributions presented in the study are included in the article/Supplementary Material, further inquiries can be directed to the corresponding author/s.

\section{REFERENCES}

Bose, A., Basu, R., Maulik, M., and Das Sarma, J. (2018). Loss of Cx43-mediated functional gap junction communication in meningeal fibroblasts following mouse hepatitis virus infection. Mol. Neurobiol. 55, 6558-6571. doi: 10.1007/ s12035-017-0861-3

Corvol, H., Rousselet, N., Thompson, K. E., Berdah, L., Cottin, G., Foussigniere, T., et al. (2018). FAM13A is a modifier gene of cystic fibrosis lung phenotype regulating rhoa activity, actin cytoskeleton dynamics and epithelialmesenchymal transition. J. Cyst. Fibros 17, 190-203. doi: 10.1016/j.jcf.2017. 11.003

Cristante, E., McArthur, S., Mauro, C., Maggioli, E., Romero, I. A., WylezinskaArridge, M., et al. (2013). Identification of an essential endogenous regulator of

\section{ETHICS STATEMENT}

The animal study was reviewed and approved by the Animal Care and Use Committee of Chongqing Medical University. Written informed consent was obtained from the owners for the participation of their animals in this study.

\section{AUTHOR CONTRIBUTIONS}

CoC drafted the manuscript and acquired funding for the study. HL, YJ, YW, JH, JZ, ZH, and ZW collected the data for this study. $\mathrm{CoC}$ and $\mathrm{CeC}$ analyzed and interpreted the data. YD edited the language of manuscript. XS formulated the study concept. $\mathrm{ZH}$ designed the study and revised the manuscript. All authors contributed to the article and approved the submitted version.

\section{FUNDING}

The research leading to these results has received funding from National Natural Science Foundation of China (No. 81571159) and National Natural Science Foundation for Youth of China (No. 81601072).

\section{ACKNOWLEDGMENTS}

We acknowledge the service provided by Chongqing Key Laboratory of Ophthalmology and Neuroprotection Research Laboratory in Boston.

\section{SUPPLEMENTARY MATERIAL}

The Supplementary Material for this article can be found online at: https://www.frontiersin.org/articles/10.3389/fnins. 2021.627110/full\#supplementary-material

\footnotetext{
Supplementary Figure 1 | The temporal changes of neutrophil infiltration following CCl. Almost no infiltrated neutrophil was seen in the sham group. The number of infiltrated neutrophils surrounding the lesion area increased maximally at day $3(p<0.05)$. Representative images and quantitative analysis of neutrophil infiltration surrounding the lesion area at the preinjury day and days 1,3 , and 7 after $\mathrm{CCl} .{ }^{*} p<0.05$ vs. sham, $n=8$ per group, scale bar $=100 \mu \mathrm{m}$.
}

blood-brain barrier integrity, and its pathological and therapeutic implications. Proc. Natl. Acad. Sci. U.S.A. 110, 832-841. doi: 10.1073/pnas.120936 2110

Delbary-Gossart, S., Lee, S., Baroni, M., Lamarche, I., Arnone, M., Canolle, B., et al. (2016). A novel inhibitor of p75-neurotrophin receptor improves functional outcomes in two models of traumatic brain injury. Brain J. Neurol. 139, 1762-1782. doi: 10.1093/brain/aww074

Donkin, J. J., and Vink, R. (2010). Mechanisms of cerebral edema in traumatic brain injury: therapeutic developments. Curr. Opin. Neurol. 23, 293-299. doi: 10.1097/WCO.0b013e328337f451

Feickert, H. J., Drommer, S., and Heyer, R. (1999). Severe head injury in children: impact of risk factors on outcome. J. Trauma 47, 33-38. doi: 10.1097/00005373199907000-00008 
Girol, A. P., Mimura, K. K., Drewes, C. C., Bolonheis, S. M., Solito, E., Farsky, S. H., et al. (2013). Anti-inflammatory mechanisms of the annexin A1 protein and its mimetic peptide Ac2-26 in models of ocular inflammation in vivo and in vitro. J. Immunol. 190, 5689-5701. doi: 10.4049/jimmunol.1202030

Heemskerk, N., Schimmel, L., Oort, C., van Rijssel, J., Yin, T., Ma, B., et al. (2016). F-actin-rich contractile endothelial pores prevent vascular leakage during leukocyte diapedesis through local RhoA signalling. Nat. Commun. 7:10493. doi: $10.1038 /$ ncomms 10493

Holmin, S., and Mathiesen, T. (2000). Intracerebral administration of interleukinlbeta and induction of inflammation, apoptosis, and vasogenic edema. J. Neurosurg. 92, 108-120. doi: 10.3171/jns.2000.92.1.0108

Huang, B., Krafft, P. R., Ma, Q., Rolland, W. B., Caner, B., Lekic, T., et al. (2012). Fibroblast growth factors preserve blood-brain barrier integrity through RhoA inhibition after intracerebral hemorrhage in mice. Neurobiol. Dis. 46, 204-214. doi: 10.1016/j.nbd.2012.01.008

Huang, Z., Cheng, C., Jiang, L., Yu, Z., Cao, F., Zhong, J., et al. (2016). Intraventricular apolipoprotein ApoJ infusion acts protectively in traumatic brain injury. J. Neurochem. 136, 1017-1025. doi: 10.1111/jnc.13491

Hubaishy, I., Jones, P. G., Bjorge, J., Bellagamba, C., Fitzpatrick, S., Fujita, D. J., et al. (1995). Modulation of annexin II tetramer by tyrosine phosphorylation. Biochemistry 34, 14527-14534. doi: 10.1021/bi00044a031

Ikebuchi, N. W., and Waisman, D. M. (1990). Calcium-dependent regulation of actin filament bundling by lipocortin-85. J. Biol. Chem. 265, 3392-3400. doi: 10.1016/S0021-9258(19)39780-7

Lee, D. B., Jamgotchian, N., Allen, S. G., Kan, F. W., and Hale, I. L. (2004). Annexin A2 heterotetramer: role in tight junction assembly. Am. J. Physiol. Renal Physiol. 287, F481-F491. doi: 10.1152/ajprenal.00175.2003

Liu, J., Wada, Y., Katsura, M., Tozawa, H., Erwin, N., Kapron, C. M., et al. (2018). Rho-associated coiled-coil kinase (ROCK) in molecular regulation of angiogenesis. Theranostics 8, 6053-6069. doi: 10.7150/thno.30305

Liu, S., Gao, Y., Yu, X., Zhao, B., Liu, L., Zhao, Y., et al. (2016). Annexin-1 mediates microglial activation and migration via the CK2 pathway during oxygen-glucose deprivation/reperfusion. Int. J. Mol. Sci. 17:1770. doi: 10.3390/ ijms 17101770

Luh, C., Feiler, S., Frauenknecht, K., Meyer, S., Lubomirov, L., Neulen, A., et al. (2019). The contractile apparatus is essential for the integrity of the bloodbrain barrier after experimental subarachnoid hemorrhage. Transl. Stroke Res. 10, 534-545. doi: 10.1007/s12975-018-0677-0

Marmarou, A. (2003). Pathophysiology of traumatic brain edema: current concepts. Acta Neurochirurgica Suppl. 86, 7-10. doi: 10.1007/978-3-70910651-8_2

McArthur, S., Loiola, R. A., Maggioli, E., Errede, M., Virgintino, D., and Solito, E. (2016). The restorative role of annexin A1 at the blood-brain barrier. Fluids Barriers CNS 13:17. doi: 10.1186/s12987-016-0043-0

Muller, W. A. (2013). Getting leukocytes to the site of inflammation. Vet. Pathol. 50, 7-22. doi: 10.1177/0300985812469883

Rao, J., Ye, Z., Tang, H., Wang, C., Peng, H., Lai, W., et al. (2017). The RhoA/ROCK pathway ameliorates adhesion and inflammatory infiltration induced by AGEs in glomerular endothelial cells. Sci. Rep. 7:39727. doi: 10.1038/srep39727

Stein, S. C., Georgoff, P., Meghan, S., Mizra, K., and Sonnad, S. S. (2010). 150 years of treating severe traumatic brain injury: a systematic review of progress in mortality. J. Neurotrauma 27, 1343-1353. doi: 10.1089/neu.2009.1206

Su, S. C., Maxwell, S. A., and Bayless, K. J. (2010). Annexin 2 regulates endothelial morphogenesis by controlling AKT activation and junctional integrity. J. Biol. Chem. 285, 40624-40634. doi: 10.1074/jbc.M110.157271

Subramanian, P., Mitroulis, I., Hajishengallis, G., and Chavakis, T. (2016). Regulation of tissue infiltration by neutrophils: role of integrin alpha3betal and other factors. Curr. Opin. Hematol. 23, 36-43. doi: 10.1097/MOH. 0000000000000198

Sugimoto, M. A., Vago, J. P., Teixeira, M. M., and Sousa, L. P. (2016). Annexin A1 and the resolution of inflammation: modulation of neutrophil recruitment, apoptosis, and clearance. J. Immunol. Res. 2016:8239258. doi: 10.1155/2016/ 8239258

Terry, S., Nie, M., Matter, K., and Balda, M. S. (2010). Rho signaling and tight junction functions. Physiology 25, 16-26.

Thomas, G., Maria, V., Anna, H., Paul, A., Stephen, M., Francesca, C., et al. (2020). Predictors of lesion cavitation after recent small subcortical stroke. Transl. Stroke Res. 11, 402-411.

Tornero, D., Wattananit, S., Gronning Madsen, M., Koch, P., Wood, J., Tatarishvili, J., et al. (2013). Human induced pluripotent stem cell-derived cortical neurons integrate in stroke-injured cortex and improve functional recovery. Brain J. Neurol. 136, 3561-3577.

Tsenter, J., Beni-Adani, L., Assaf, Y., Alexandrovich, A. G., Trembovler, V., and Shohami, E. (2008). Dynamic changes in the recovery after traumatic brain injury in mice: effect of injury severity on T2-weighted MRI abnormalities, and motor and cognitive functions. J. Neurotrauma 25, 324-333.

Wang, Z., Higashikawa, K., Yasui, H., Kuge, Y., Ohno, Y., Kihara, A., et al. (2020). FTY720 protects against ischemia-reperfusion injury by preventing the redistribution of tight junction proteins and decreases inflammation in the subacute phase in an experimental stroke model. Transl. Stroke Res. 11, 1103-1116. doi: 10.1007/s12975-020-00789-x

Wright, D. W., Yeatts, S. D., Silbergleit, R., Palesch, Y. Y., Hertzberg, V. S., Frankel, M., et al. (2014). Very early administration of progesterone for acute traumatic brain injury. N. Engl. J. Med. 371, 2457-2466.

Wu, J., He, J., Tian, X., Zhong, J., Li, H., and Sun, X. (2020). Activation of the hedgehog pathway promotes recovery of neurological function after traumatic brain injury by protecting the neurovascular unit. Transl. Stroke Res. 11, 720-733. doi: 10.1007/s12975-019-00771-2

Zemmar, A., Weinmann, O., Kellner, Y., Yu, X., Vicente, R., Gullo, M., et al. (2014). Neutralization of Nogo-A enhances synaptic plasticity in the rodent motor cortex and improves motor learning in vivo. J. Neurosci. 34, 8685-8698.

Zhang, C., Jiang, M., Wang, W. Q., Zhao, S. J., Yin, Y. X., Mi, Q. J., et al. (2019). Selective mGluR1 negative allosteric modulator reduces blood-brain barrier permeability and cerebral edema after experimental subarachnoid hemorrhage. Transl. Stroke Res. 11, 799-811. doi: 10.1007/s12975-019-00 758-z

Zhang, W., Zhang, H., Mu, H., Zhu, W., Jiang, X., Hu, X., et al. (2016). Omega3 polyunsaturated fatty acids mitigate blood-brain barrier disruption after hypoxic-ischemic brain injury. Neurobiol. Dis. 91, 37-46.

Zhao, S., Yu, Z., Liu, Y., Bai, Y., Jiang, Y., van Leyen, K., et al. (2017). CD47 deficiency improves neurological outcomes of traumatic brain injury in mice. Neurosci. Lett. 643, 125-130.

Conflict of Interest: The authors declare that the research was conducted in the absence of any commercial or financial relationships that could be construed as a potential conflict of interest.

Copyright (c) $2021 \mathrm{Liu}, \mathrm{He}, \mathrm{Wu}$, Du, Jiang, Chen, Yu, Zhong, Wang, Cheng, Sun and Huang. This is an open-access article distributed under the terms of the Creative Commons Attribution License (CC BY). The use, distribution or reproduction in other forums is permitted, provided the original author(s) and the copyright owner(s) are credited and that the original publication in this journal is cited, in accordance with accepted academic practice. No use, distribution or reproduction is permitted which does not comply with these terms. 